\title{
Blind People and Mobile Keypads: Accounting for Individual Differences
}

\author{
Tiago Guerreiro, João Oliveira, João Benedito, Hugo Nicolau, \\ Joaquim Jorge, and Daniel Gonçalves \\ INESC-ID / Technical University of Lisbon, Portugal \\ \{tjvg, hman, jaj, djvg\}@immi.inesc-id.pt, \\ \{jmgdo, jpmlb\} @ist.utl.pt
}

\begin{abstract}
No two persons are alike. We usually ignore this diversity as we have the capability to adapt and, without noticing, become experts in interfaces that were probably misadjusted to begin with. This adaptation is not always at the user's reach. One neglected group is the blind. Age of blindness onset, age, cognitive, and sensory abilities are some characteristics that diverge between users. Regardless, all are presented with the same methods ignoring their capabilities and needs. Interaction with mobile devices is highly visually demanding which widens the gap between blind people. Herein, we present studies performed with 13 blind people consisting on key acquisition tasks with 10 mobile devices. Results show that different capability levels have significant impact on user performance and that this impact is related with the device and its demands. It is paramount to understand mobile interaction demands and relate them with the users' capabilities, towards inclusive design.
\end{abstract}

Keywords: Individual Differences, Mobile Accessibility, Blind, Mobile Device, User Assessments.

\section{Introduction}

Mobile devices have become indispensable tools in our daily lives and are now used by everyone in several different situations. However, every human is different and so is every situation. This diversity has not been given enough attention in mobile user interface design decreasing the user's effectiveness or even hindering the ability to interact. Particularly, disabled target groups, characterized by specific individual differences, can greatly benefit from an effective mobile user interface [1]. However, alternative user interfaces are likely to be misaligned with the users and their identities. These adapted mobile user interfaces are stereotypical disregarding that the relation between the user and the device depends on particular characteristics and not on a common idea. In general, mobile interaction has not evolved to meet the users' requirements.

We focus our attention on blind people. The absence of such an important and integrating sense as vision, in the presence of so demanding interfaces as are the ones in current mobile devices, justifies it. Besides personality differences, two blind users 
are likely to have totally different stories to what blindness, and its implications, is concerned. The cause of the impairment, age of onset, time with impairment, age, simultaneous impairments, cognitive or sensorial abilities, are some examples of the characteristics that may diverge between users. Some of these may implicate others, some may be collateral damages, and others can overcome some. A young 'recentblind' is different from an older one. While the former is likely to have all his other senses immaculate, the latter may have some other age-related impairments. However, he is also likely to have developed sensory compensation mechanisms. How are they different and how will those differences affect their functional ability?

What is indisputable is that the enormous diversity found among this particular group of users turns the "stereotypical blind" idea inadequate. As an example, age, its related degenerations, but also how it influences sensory compensation and augments individual differences, is an example of a characteristic that must be considered when discussing a particular blind person. As blindness age of onset can determine how one will face the challenges imposed by any daily task. These characteristics have a huge impact on the blind users' lives and how they are able to deal with technology. Regardless, all are presented with the same methods and opportunities ignoring their capabilities and needs.

Interaction with mobile devices is highly visually demanding which increases the difficulties. Even mobile assistive technologies for the blind have a narrow and stereotypical perspective over the difficulties faced by their users. A blind user is presented with screen reading software to overcome the inability to see onscreen information. However, these solutions go only half-way. In the absence of sight other aptitudes/limitations stand up. To empower these users, a deeper understanding of their capabilities and how they relate with technology is mandatory. As such, we performed a study with the target population consisting on key acquisition tasks with different mobile keypads, in order to relate individual differences with devices' demands.

\subsection{The Blind}

We focus on a particular target group: blind people. This can be explained both by the high visual demands imposed by current mobile device interfaces (which are increasing, e.g., touch screens) and the diversity within the population. In this section, we offer an overview of the population and related concepts, valuable to understanding the remaining of this paper.

\subsubsection{Causes}

Blindness is due to a variety of causes. Age-related blindness is increasing throughout the world, as is blindness due to uncontrolled diabetes. Diabetes is responsible for $8 \%$ of legal blindness, according to the American Diabetes Association, making it the leading cause of new cases of blindness in adults 20-74 years of age. This is significant since diabetic retinopathy is often accompanied by peripheral neuropathy which also impairs the sense of touch [2]. 


\subsubsection{Worldwide Statistics}

The American Foundation for the Blind estimates that there are 10 million blind or visually impaired people in the United States. In a survey realized in 1994-1995, 1.3 million Americans (0.5\%) reported being legally blind. Of this number, only $10 \%$ were totally blind and another $10 \%$ had only light perception. The remaining $80 \%$ had some useful vision. Few statistics appear to be available about the age of onset of blindness. It is reported that only eight percent of visually impaired people are born with any impairment [3]. Worldwide, an estimated 180 millions are visually impaired, of which 40-45 millions are blind [4].

The prevalence of blindness is much higher for the elderly [2]. It is estimated that $1.1 \%$ of the elderly (65 and over) are legally blind compared to $0.055 \%$ of the young (20 and under) [5]. About $82 \%$ of all people who are visually impaired are age 50 and older (although they represent only $19 \%$ of the world's population). The attitude towards blindness as well as space representation may be affected by the age of onset of blindness [2]. It is also reported that more than 50 percent of individuals with visual impairments also have one or more other impairments [6]. It is worth mentioning that blindness is expected to increase in the following years [5].

\subsubsection{Individual Differences among Blind People}

Previous studies [7] have shown that individual differences among blind people are likely to have a wider impact on their abilities to interact with mobile devices than among sighted people. Tactile sensitivity, spatial ability, short-term memory, blindness onset age and age are mentioned as deciding characteristics for a blind user mobile performance.

The capability-demand theory builds on the concepts of user capability and product demand and aims to analyze user-product compatibility, i.e. an assessment and comparison of the sensory, cognitive and motor demand made by a product in relation to the ability levels of the expected user population [8]. We embrace the capabilitydemand theory and aim to assert relations between users and devices, and ultimately aim at a match between individual capabilities and mobile interaction demands. This way, we will provide both the tools for mobile designers, showing which designs are most effective and inclusive, and for blind users, identifying for each one, the most appropriate interfaces.

\section{Related Work}

Mobile interaction is still in its early stages when compared with interaction with desktop computers that has been subject of attention for several decades. Although mobile computing is an active research theme, mobile HCI was not until recently an important subject. In particular, only few researchers have leaned over the multitude of individuals, scenarios and situations faced by mobile devices.

The related work presented here is twofold. Firstly, we survey mobile user interfaces for blind users and present the actual research state considering alternative interfaces for the target population. Secondly, we present projects that have focused on 
individual differences, taking into consideration some kind of particular characteristics instead of a global "average user" model.

\subsection{Mobile Interaction for the Blind}

A blind person, or even one with low vision, faces several limitations when interacting with mobile devices $[9,10,11]$. Looking at these, whether keypad or touch screenbased, the interaction mechanisms are convoluted to deal with the limited input area and overall device small size. Furthermore, the mechanisms found to overcome the lack of space (when compared to desktop computers) resort to an intensive visualbased dialogue with the mobile device user. As an example, several text-entry systems are based on multi-tap approaches where the user is able to see both the relation between keys and letters (visual feedback from the physical or virtual keys), and the evolution of the process on the display. A user with severe visual limitations is unable to receive this information and thus his ability to interact with these devices is highly limited.

An attempt to attenuate difficulties arising from disabilities is being made through assistive technologies, designing new solutions based on alternative interaction methods such as haptic interfaces, screen readers or multimodal information feedback [12]. In the case of mobile phones, accessibility solutions consist of devices with native features to make them more accessible to a given population, the so called accessible phones, or mobile phones that allow third-party software installation like screen readers or screen magnifiers.

Special mobile devices were developed to overcome the barriers arising from visual impairments. As examples are the Braillino or the Alva Mobile Phone Organizer, among many others very similar between each other [13]. These devices, which typically work as a Personal Digital Assistant, or as a docking station, use a Braille keyboard for text input, a Braille screen for output information, and provide functionalities like the ones provided in regular mobile phones. Yet, they all share the same flaws: their cost is prohibitive and they are not as portable as a mobile phone is, being too big and heavy. Even though their cost and size has decreased, they are still not as practical as common mobile devices and have the inconvenient to "look disabled". Another factor, and one of the most important, is that although Braille is the recognized alternative language for blind people, a reduced percentage is actually Brailleknowledgeable ${ }^{1}$.

Nowadays, a common mobile solution for blind users resorts to the usage of a screen reader, replacing the visual feedback by its auditory representation (e.g., Mobile Speak ${ }^{2}$ or Nuance Talks ${ }^{3}$ ) [14]. This approach supposedly enables the users to access the same applications a fully-sighted user accesses. The ability to use a "nondisabled" device with the same characteristics (technical, social and economical) is a great advantage of this type of solutions. However, the offered feedback is restricted

\footnotetext{
${ }^{1}$ American Foundation for the Blind: Programs and Policy Research, "Estimated Number of Adult Braille Readers in the United States", International Braille Research Center (IBRC), http://www.braille.org/papers/jvib0696/vb960329.htm

${ }^{2} \mathrm{http}: / / \mathrm{www}$. codefactory.es/en/products.asp?id=316

${ }^{3} \mathrm{http} / / / \mathrm{www}$.nuance.com/for-individuals/by-solution/talks-zooms/index.htm
} 
to the output, as no information is obtained on key/function relation. Moreover, the information on the screen is prepared for visual feedback and not to be read. As an example, considering text-entry, screen reader approaches force the user to try to find the desired letter in the keypad, committing several errors in the process, and possibly leading to situations where he/she simply quits trying. A person that acquires blindness in an advanced stage of life, along with the reduction of other capabilities such as tactile sensitivity, is likely to face difficulties in the first contact with this approach, rejecting it before gaining the experience that enables its use [9]. In contrast to traditional interfaces, designed for the "average user", simple screen reading approaches are designed for the "stereotypical blind", one that has improved tactile and auditory senses along with good mental health and motivation.

Guerreiro et al. [15] proposed two non-visual texting interfaces for keypad-based devices - NavTap and BrailleTap - that take advantage of blind users' capabilities. NavTap was designed for those who are not able to learn traditional MultiTap methods, allowing them to easily navigate through the alphabet using only four keys, thus eliminating the need to memorize mappings between keys and letters. Similarly, by transforming the traditional keypad layout into a more familiar interface, BrailleTap was designed for those who master the Braille alphabet, allowing them to enter text on common devices. More recently, with the emergence of touch screen devices, several approaches have been proposed featuring directional gestures for menu navigation [16] and text-entry tasks $[17,18]$.

In general, there has been as effort to provide visually impaired users with technologies that are able to surpass the target group's problems and inabilities. However, the majority of the approaches are designed taking into consideration a "stereotypical" image of the blind user. Whether considering Braille interfaces or other touchdemanding interfaces, they are created without a real knowledge of the users and their needs. If blind-targeted interfaces were designed following an user-centered design approach, nowadays they would not be merely based on the Braille alphabet (as the overall knowledge is minimum) nor on the users' tactile capabilities (as a great majority of the blind population are older adults with no compensatory mechanisms and low tactile capabilities). However, there are interfaces characteristics that have shown to be advantageous for particular sets of users. Further research needs to be performed to assess the individual differences within the target population and understand the interface demands and match for particular blind users.

\subsection{Accounting for Individual Differences}

While desktop computers already offer a multitude of personalization capacities, whether considering individual differences or just taste, mobile devices are still restricted to a limited set of personalization options, which are majorly aesthetic or related to the users' personality. A better understanding of the individual differences that characterize the users in the mobile interaction context is required. Only with that knowledge will we be able to "prescribe" adequate devices and interfaces that empower the users.

While it is important to understand that a mobile device user is different from the next one and that those differences should be considered to improve device accessibility, it is also important to understand that, even for a single user, his capacities and 
needs are likely to diverge across time (dynamic diversity) [19]. Gregor and Newell state that most computer systems are designed for a typical younger user with static abilities over time. However, even when user-centered paradigms are employed, the designers look typically at concerns such as representative user groups, without regard for the fact that the user is not a static entity. This does not take into account the wide diversity of abilities among users and it also ignores the fact that these abilities are dynamic over time. The authors propose a new paradigm, designing for Dynamic Diversity, based on a user-sensitive inclusive design methodology [19]. The use of the term "inclusive" rather than "universal" reflects the view that "inclusivity" is a more achievable, and in many situations, appropriate goal than "universal design" or "design for all". "Sensitive" replaces "centered" to underline the extra levels of difficulty involved when the range of functionality and characteristics of the user groups can be so great that it is impossible in any meaningful way to produce a small representative sample of the user group nor often to design a product which truly is accessible by all potential users [19].

In order to lessen the difficulties that many people feel when interacting with mobile devices, inclusive design when developing these products is crucial. To make this a reality, it is necessary to attend to the many different characteristics of the user. Persad et al. [8] propose an analytical evaluation framework based on the CapabilityDemand theory, where user capabilities at sensory, cognitive and motor levels, are matched with product demands.

When studying interfaces for blind people, a capability that should not be ignored is tactile sensibility. Besides being crucial to capture information at the expense of vision, approximately $82 \%$ of all people who are blind are aged 50 or more [20] and as diabetes is one the main causes of blindness, changes in this sensorial capability are fairly common and should be accounted for. In [21,22] several physical requirements were identified in order for mobile devices to be accessible with limited sensibility. Despite the fact that these studies acknowledged key requirements, these characteristics were not quantified nor related with the different users' abilities.

Cognitive capabilities such as short-term memory, attention and spatial ability should also be meaningful when developing interfaces for the blind. Mobile interaction requires a cognitive effort that, for someone lacking sight, is much more demanding. Although there are studies that relate cognitive ability with mobile device usage for sighted older adults [23], there is an enormous gap in terms of studies relating cognitive ability and mobile phone interaction of a visually impaired person.

\section{Assessing the Impact of Individual Differences}

Mobile accessibility solutions for blind users are commonly presented as audio replacements for onscreen information. However, there is more to it. Not only the interaction is much more demanding in the absence of sight, but also blind people often present differences that make them far in capability from the stereotyped blind. 


\subsection{Research Goals}

In this study, we intend to understand the impact of individual differences among the blind when interacting with mobile device keypads as well as how these differences are revealed when confronted with different device demands. Particularly, we want to answer the following research questions: 1) Does tactile sensitivity affect performance on a simple key acquisition task?; 2) Does cognitive ability affect performance on a simple key acquisition task?; 3) Which keyboard demands have most impact on user performance?; 4) Are individual differences worth considering on mobile interface design?

\subsection{Procedure}

To evaluate the match between users and interfaces by using various measures of compatibility, we performed studies with the target population. They were threefold. The first two consisted in measuring user capabilities, more specifically, tactile sensitivity and cognitive ability. In the third and final, we conducted an experimental task to assess user performance in a simple key acquisition task. Details on each of these studies and the results obtained considering the different capabilities and product demands are depicted in the following sections.

\subsection{Tactile Sensitivity}

To assess the participants' tactile capabilities, two different components of tactile sensitivity were measured. The first, pressure sensitivity, was determined using the Semmes-Weinstein monofilament test [24] (Figure 1). In this test, there are several nylon filaments with different levels of resistance, bending when the maximum pressure they support is applied. This way, if a user can sense a point of pressure, his pressure sensitivity is equal to the force applied by the filament.
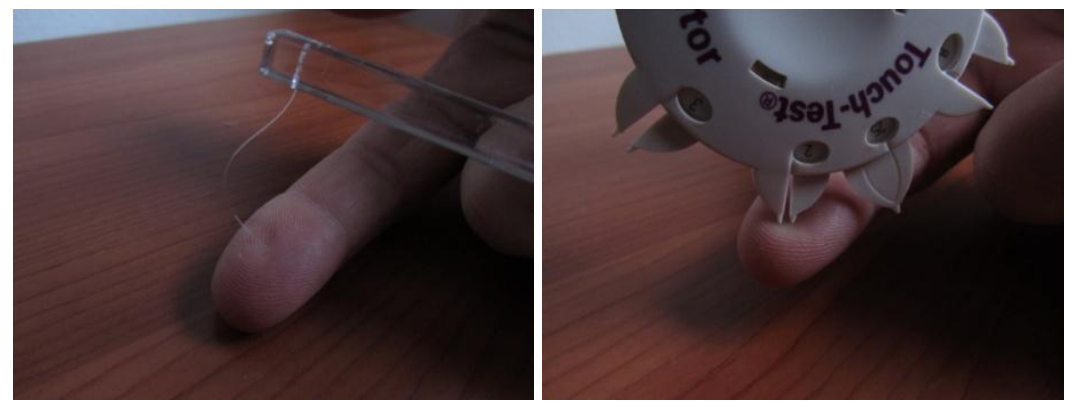

Fig. 1. Tactile assessment: Semmes-Weinstein test (left) and Disk-Criminator (right)

Five monofilaments of 2.83, 3.61, 4.31, 4.56 and 6.65 Newton were used, starting the stimuli with the one of 2.83 , the least resistant one. Pressure was applied in the thumb, index and middle fingers, those generally used when interacting with mobile 
devices, and in random order, so we could prevent arbitrary identification of a stimulus by the person being tested. The process is repeated with the filament with the next resistance level, until all filaments are tested or the participant correctly identifies the stimulus made. Different levels can be found for different fingers.

The other tactile sensitivity component measured was spatial acuity, using the Disk-Criminator [25] (Figure 1). This instrument measures a person's capability to distinguish one or two points of pressure on the skin surface. The Disk-Criminator is generally an orthogonal plastic instrument that has in each side a pair of metal filaments with relative distances ranging from 1 to $25 \mathrm{~mm}$. When the person being tested identifies a stimulus as being two points, her spatial acuity discrimination is equal to the distance between the filaments.

The distance between the filaments of the Disk-Criminator tested ranged from 2 to $15 \mathrm{~mm}$, with $1 \mathrm{~mm}$ increments. Each of these filament pairs was, as with the pressure sensitivity, applied randomly in the same three fingers. There were made 10 stimuli per finger, randomly, alternating between a pair of filaments and a unique filament. The participant had to indicate when he/she felt one or two points of pressure. When he/she was able to correctly identify 7 out of 10 stimuli, his/her level of spatial acuity was registered as the distance between filaments.

\subsection{Cognitive Assessment}

The cognitive evaluation focused two components of the cognitive ability, a verbal and a non-verbal. The verbal component was evaluated in terms of working memory: a short-term memory and main responsible for the control of attention [26]. The nonverbal component, which consists of abilities independent of mother language or culture, was evaluated in terms of spatial ability: the ability to create and manipulate mental images, as well as maintain orientation relatively to other objects [27].

To evaluate working memory, the subtest Digit Span of the revised Wechsler Adult Intelligence Scale (WAIS-R) was used [28]. In the first part of this test, the participant must repeat increasingly long series of digits presented orally, and on the second, repeat other sets of numbers but backwards. The last number of digits of a series properly repeated allows calculation of a grade to the participant's working memory and, subsequently, to the user's verbal intelligence quotient (Verbal IQ).
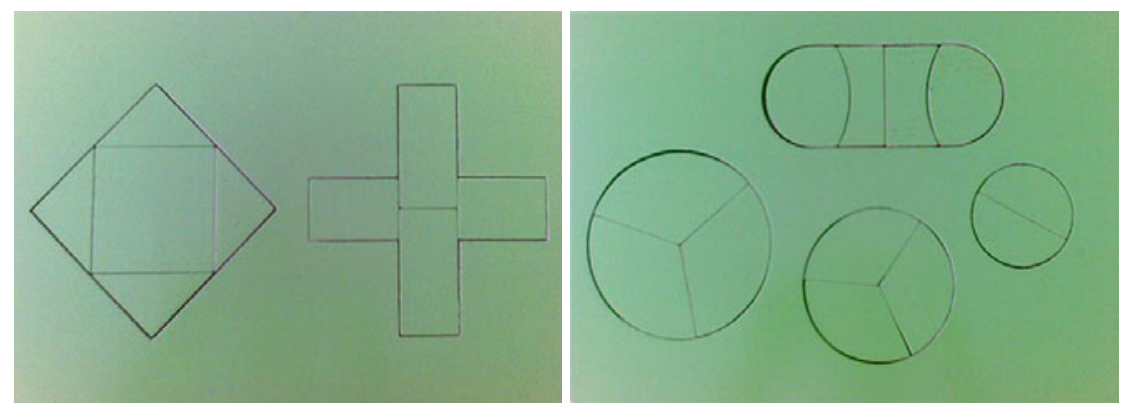

Fig. 2. Spatial ability test: Planche a Deux Formes (left) and Planche du Casuiste (right) 
Spatial ability was measured using the combined grades of the tests Planche a Deux Formes and Planche du Casuiste. These two tests are part of a cognitive battery for vocational guidance [29]. The goal of these tests is to complete, as fast as possible, a puzzle of geometrical pieces (Figure 2).

A K-means algorithm was used to group the Spatial Ability and Verbal IQ values into levels according to their different measures. The different values were grouped into three levels: Inferior, Average, Superior (Table 1).

Table 1. The studied cognitive ability characteristics and the group levels formed with a KMeans algorithm. Each group level's measure corresponds to the cluster's centre value.

\begin{tabular}{|l|c|c|c|l|}
\hline \multirow{2}{*}{\multicolumn{1}{|c|}{ Attribute }} & \multicolumn{3}{c|}{ Group Levels } & \multirow{2}{*}{ Measure } \\
\cline { 2 - 4 } & Inferior & Average & Superior & \\
\hline Spatial Ability & 1.90 & 5.20 & 9.03 & Spatial ability score (1-20). \\
\hline Verbal IQ & 72 & 91 & 129 & Verbal IQ score. \\
\hline
\end{tabular}

\subsection{Experimental Task}

While we acknowledge that the challenges imposed to blind users are spread among different interaction layers, we focused our attention in the first barrier they encounter, the physical one. Therefore, we chose the simplest task when using a mobile device, so we could isolate its demands and understand their effect on users' performance. The task presented in this study consisted of simple key acquisitions with 10 different mobile devices (Figure 3). All selected phones featured a keypad, since they are still the most used among the blind. None of them had any sort of accessibility aid. Devices were categorized according to their keypads' physical attributes. A Kmeans algorithm was used to group these attributes into levels according to their different measures. Key Spacing and Key Height values were grouped into three levels: Smaller, Average and Bigger; while Key Size and Key Label into two: Smaller and Bigger. The values of the last attribute Key Material were grouped into two categories: Good, if the materials on the phone and keyboard are distinct, and Bad if not. Table 2 shows the keyboard attributes and their groups, including the value of the clusters' center for each level.

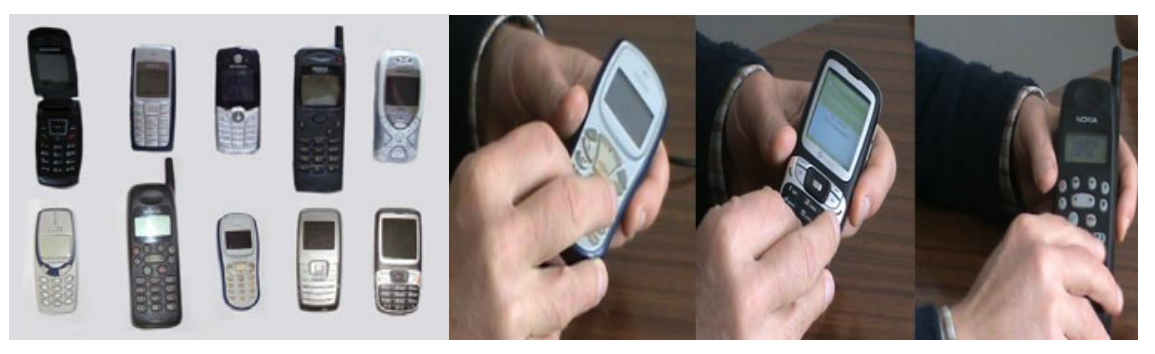

Fig. 3. Mobile phones tested (from top left to bottom right: Samsung C108, Nokia 1110, Motorola C650, Nokia 6150, Siemens MC 60, Nokia 3310, Nokia 638, Sendo, Samsung, HTC $\mathrm{S} 310$ ). A test with a user in three of the ten mobile devices (right). 
Our goal in this experimental task was to evaluate performance, in terms of efficiency (time) and effectiveness (number of errors), in low level tasks. Each participant was asked, randomly, to input a key (0 to 9, “*” and “\#”) with the keypad. All numbers were issued two times making a total of 24 keys by phone (Figure 3 ). After each acquisition, the participant had to place the operating hand resting on the table.

All of the mobile devices were shown, before the evaluation, to the volunteers, so they could experiment and understand the layout. All doubts were cleared by the researchers.

The time taken to press each key was measured (Average Time) as well as the number of errors (Task Errors), with an error being considered when the participant pressed the wrong key, pressed more than a key, or tried to press a key but failed.

Table 2. The keyboards' attributes and the group levels formed with a K-Means algorithm. Each group level's measure corresponds to the cluster's center value.

\begin{tabular}{|c|c|c|c|c|c|}
\hline \multirow{2}{*}{\multicolumn{2}{|c|}{ Attribute }} & \multicolumn{3}{|c|}{ Group Levels } & \multirow{2}{*}{ Measure } \\
\hline & & Smaller & Average & Bigger & \\
\hline \multirow{2}{*}{$\begin{array}{l}\text { Key } \\
\text { Spacing }\end{array}$} & Horizontal & $0.7 \mathrm{~mm}$ & $2.2 \mathrm{~mm}$ & $4.6 \mathrm{~mm}$ & \multirow{2}{*}{$\begin{array}{l}\text { Distance between the closest edges } \\
\text { of the keys, horizontally and verti- } \\
\text { cally ( } \mathrm{mm} \text { ). }\end{array}$} \\
\hline & Vertical & $0.8 \mathrm{~mm}$ & $2.2 \mathrm{~mm}$ & $3.3 \mathrm{~mm}$ & \\
\hline $\begin{array}{l}\text { Key } \\
\text { Height }\end{array}$ & & $0 \mathrm{~mm}$ & $0.5 \mathrm{~mm}$ & $1.1 \mathrm{~mm}$ & $\begin{array}{l}\text { Distance between the base and the } \\
\text { top of the key }(\mathrm{mm}) \text {. }\end{array}$ \\
\hline \multirow{2}{*}{ Key Size } & Horizontal & $8.3 \mathrm{~mm}$ & & $11.7 \mathrm{~mm}$ & \multirow{2}{*}{$\begin{array}{l}\text { Largest side or diameter of the } \\
\text { polygon, horizontally and vertically } \\
(\mathrm{mm}) \text {. }\end{array}$} \\
\hline & Vertical & $4.8 \mathrm{~mm}$ & & $6.1 \mathrm{~mm}$ & \\
\hline $\begin{array}{l}\text { Key } \\
\text { Label }\end{array}$ & & $0.1 \mathrm{~mm}$ & & $0.4 \mathrm{~mm}$ & $\begin{array}{l}\text { Height of the label(s) on the num- } \\
\text { ber } 5 \text { key }(\mathrm{mm}) \text {. }\end{array}$ \\
\hline $\begin{array}{l}\text { Key } \\
\text { Material }\end{array}$ & & & & & $\begin{array}{l}\text { If the key material is contrasting } \\
\text { with the mobile phone's base mate- } \\
\text { rial. }\end{array}$ \\
\hline
\end{tabular}

\subsection{Participants}

The participant group was composed by 13 students ( 8 female, 5 male) from a formation centre for visually impaired people, where all the evaluations took place. With ages ranging from 25 to 61 years old (averaging 50), all of the volunteers were blind (at most light perception).

All of the participants used mobile phones on a daily basis with the help of screen readers. However, even when using screen readers, 3 participants stated that they have difficulty sending text messages, restricting use to placing and receiving calls. Their characterization, including the results of the tactile sensitivity and cognitive assessments, is displayed in Table 3.

\subsection{Design and Analysis}

This study features two dependent variables (Average Time and Task Errors) and several factors related to device attributes (Key Size, Key Spacing, Key Height, Key Material and Key Label) as well as participants' individual differences (Blindness Onset, Time with Impairment, Education, Spatial Acuity, Pressure Sensitivity, Spatial Ability, Verbal IQ). 
Table 3. Participant's characterization. The lower the spatial acuity and pressure sensitivity values, the better the tactile sensitivity. The opposite for verbal IQ and spatial ability.

\begin{tabular}{lcccccccc}
\hline User & Gender & Age & $\begin{array}{c}\text { Time with } \\
\text { Impairment }\end{array}$ & Education & $\begin{array}{c}\text { Spatial } \\
\text { Acuity }\end{array}$ & $\begin{array}{c}\text { Pressure } \\
\text { Sensitivity }\end{array}$ & $\begin{array}{c}\text { Verbal } \\
\text { IQ }\end{array}$ & $\begin{array}{c}\text { Spatial } \\
\text { Ability }\end{array}$ \\
P01 & Female & 48 & 2 Years & $4^{\text {th }}$ Grade & 3 & 4.31 & 66 & 1,75 \\
P02 & Female & 58 & 54 Years & $6^{\text {th }}$ Grade & 4 & 3.61 & 69 & 1,75 \\
P03 & Female & 53 & 21 Years & $7^{\text {th }}$ Grade & 3 & 4.31 & 84 & 3,25 \\
P04 & Female & 48 & 31 Years & $9^{\text {th }}$ Grade & 2 & 4.31 & 78 & 5,5 \\
P05 & Female & 39 & 5 Years & $9^{\text {th }}$ Grade & 2 & 4.31 & 79 & 7,75 \\
P06 & Male & 61 & 58 Years & $6^{\text {th }}$ Grade & 2 & 4.31 & 104 & 4,75 \\
P07 & Male & 25 & 6 Years & $12^{\text {th }}$ Grade & 2 & 2.83 & 64 & 5,5 \\
P08 & Female & 53 & 33 Years & $4^{\text {th }}$ Grade & 4 & 4.31 & 78 & 1 \\
P09 & Male & 51 & 16 Years & $9^{\text {th }}$ Grade & 3 & 4.31 & 84 & 6,25 \\
P10 & Male & 59 & 59 Years & $9^{\text {th }}$ Grade & 2 & 4.31 & 134 & 4 \\
P11 & Female & 41 & 21 Years & $12^{\text {th }}$ Grade & 2 & 3.61 & 123 & 10,85 \\
P12 & Male & 58 & 24 Years & $9^{\text {th }}$ Grade & 2 & 4.31 & 86 & 8,5 \\
P13 & Female & 55 & 55 Years & $10^{\text {th }}$ Grade & 2 & 3.61 & 98 & 1,75 \\
\hline
\end{tabular}

Shapiro-Wilk test was used to examine the normality of the data. Since the two dependent variables did not exhibit a normal distribution, Kruskal-Wallis test was used to assess significant differences of the different independent variables. When statistically analysing multiple factors, a two-way ANOVA was used, admitting data normality. Correlation between Blindness Onset and Time with Impairment with the dependent variables was measured using Pearson's Correlation Coefficients.

\section{Results}

Our goal is to understand the impact of each characteristic and how these differences are revealed when subject to different demands. First, we focus on each user attribute and present the impact on overall performance. Then, we look into device demand variations and observe how they affect participants' effectiveness and efficiency. Hence, we will be able not only to call researchers and manufactures attention to individual differences but also to point out the demands that should be considered to promote inclusive design.

\subsection{Individual Differences}

Blindness Onset age had a positive low degree correlation with Task Errors $(r=0.22$, $p<.05)$. It also had significant moderate correlation with Average Time $(r=0.29$, $p<.01$ ). The greater the age of onset, the less time and number of errors the users made. A relation between Time with Impairment and Average Time was also found. The bigger the Time with Impairment, the less Average Time the users took, as a moderate negative correlation was found $(r=0.37, p<.01)$.

The user's Education had an impact on the_Task Errors $\left(\mathrm{X}^{2}(2)=8.34, p<.01\right)$. More educated user's made significantly fewer errors on the task proposed. 
In terms of tactile sensitivity, Spatial Acuity had a significant effect on Average Time $\left(\mathrm{X}^{2}(1)=4.204, p<.05\right)$. Participants with better spatial acuity $(2 \mathrm{~mm})$ were faster than the remaining (Figure 4).

Regarding cognitive ability, Spatial Ability did not impact the efficiency or the effectiveness of the volunteers. On the other hand, the Verbal $I Q$ relation with Average Time and Task Errors had a significant effect $\left(\mathrm{X}^{2}(2)=21.92, p<.01\right.$ and $\mathrm{X}^{2}(2)=6.33$, $p<.05$ ). The higher the Verbal IQ, less time the users took to press a button (Figure 4), while also committing fewer errors (Figure 5). Although Spatial Ability has not shown significant effects, the two cognitive abilities together had a significant influence on the outcome of the user's performance $\left(F_{3,122}=5.63, p<.01\right)$.
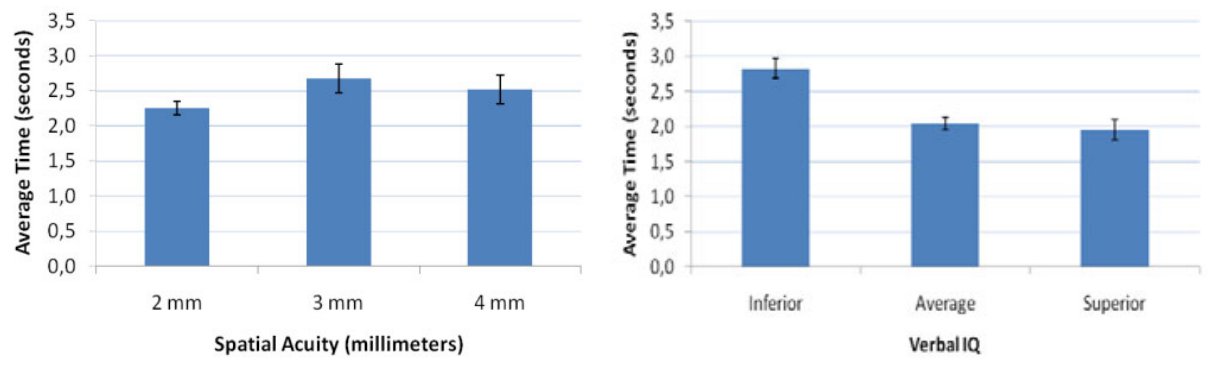

Fig. 4. Average Time for Spatial Acuity (left) and Verbal IQ (right). Error bars denote 95\% CI.
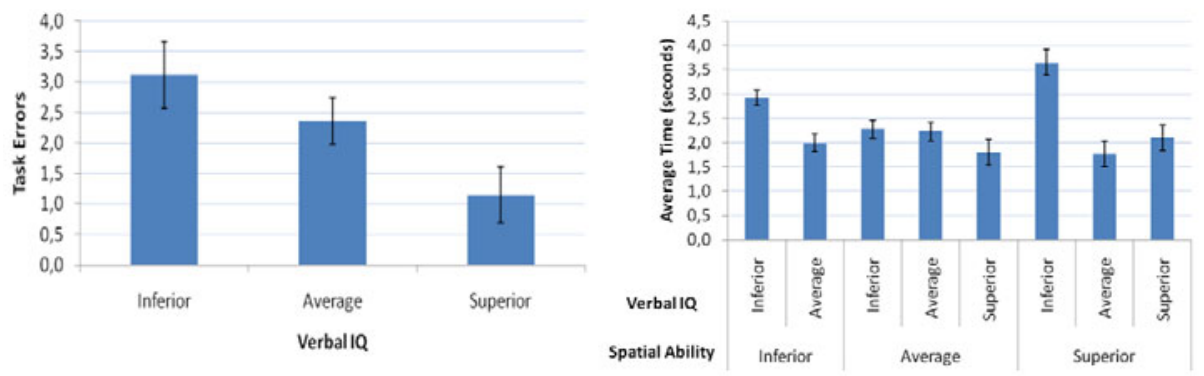

Fig. 5. Task Errors (average) for Verbal IQ (left) and Average Time for Spatial Ability and Verbal IQ (right). Error bars denote $95 \%$ CI.

Looking at the relation of cognitive ability with tactile sensibility, a multiple factor analysis shows that the Verbal IQ and Spatial Acuity affected significantly the efficiency and effectiveness of the participants $\left(F_{1,124}=4.20, p<.05\right.$ and $F_{1,124}=8.85, p<.01$ respectively). Figure 6 shows that for participants with the same Spatial Acuity, performance improved with Verbal $I Q$ increase. 

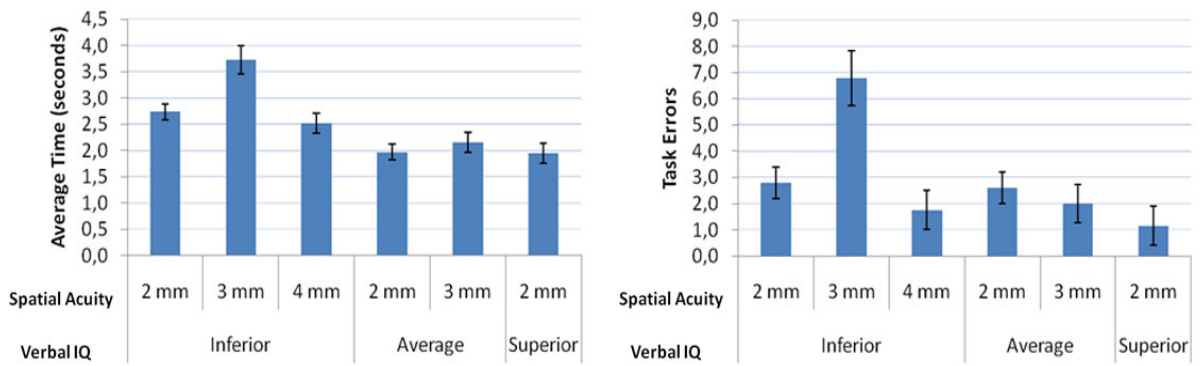

Fig. 6. Average Time for each Verbal IQ and Spatial Acuity (left) and Task Errors (average) for each Verbal IQ and Spatial Acuity (right). Error bars denote 95\% CI.

\subsection{Mobile Devices}

The analysis of the participants' performance on each mobile phone shows that the number of errors and time varies across all devices (Figure 7). The analysis of variance shows that the devices' attributes influence significantly the users' efficiency $\left(\mathrm{X}^{2}(9)=39.81, p<.01\right)$ and effectiveness $\left(\mathrm{X}^{2}(9)=46.72, p<.01\right)$.
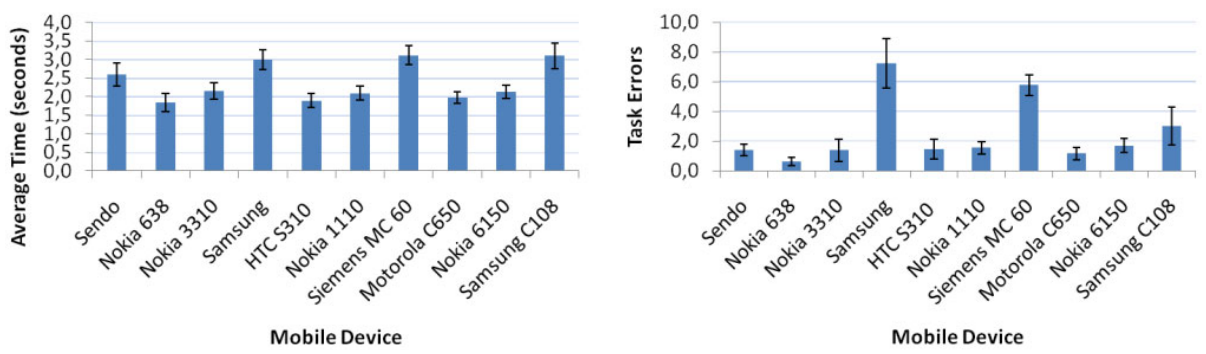

Fig. 7. Average Time for each Mobile Device (left) and Task Errors (average) for each Mobile Device (right). Error bars denote 95\% CI.

If we look at each attribute by itself, all of them, with the exception of Key Material, had a significant effect on Task Errors $\left(\mathrm{X}^{2}(1)=7.68, p<.01\right.$ for Key Size, $\mathrm{X}^{2}(2)=13.26, p<.01$ for Key Height, $\mathrm{X}^{2}(2)=10.52, p<.01$ for Key Spacing and $\mathrm{X}^{2}(1)=7.28, p<.01$ for Key Label). As we can see in Figure 8, the bigger and more pronounced the attribute was, fewer errors participants made.

The relation of the attribute Key Height with Average Time was also significant $\left(\mathrm{X}^{2}(2)=16.12, p<.01\right)$, as we can see on Figure 9.

A multiple factor analysis between the different characteristics of the mobile devices revealed a couple of significant pairs. The pair Key Size and Key Height had a significant effect on Average Time and Task Errors of the participants $\left(F_{2,124}=14.47\right.$, $p<.01$ and $F_{2,124}=2.84, p<.01$ respectively). We can verify from Figures 9 and 10 that participants made by far most mistakes and took the longest time when interacting 
with devices with a small Key Spacing and an average Key Size. It is also interesting to note that a better performance resulted from a higher Key Spacing between keys in conjunction with average Key Size, than with the opposite.
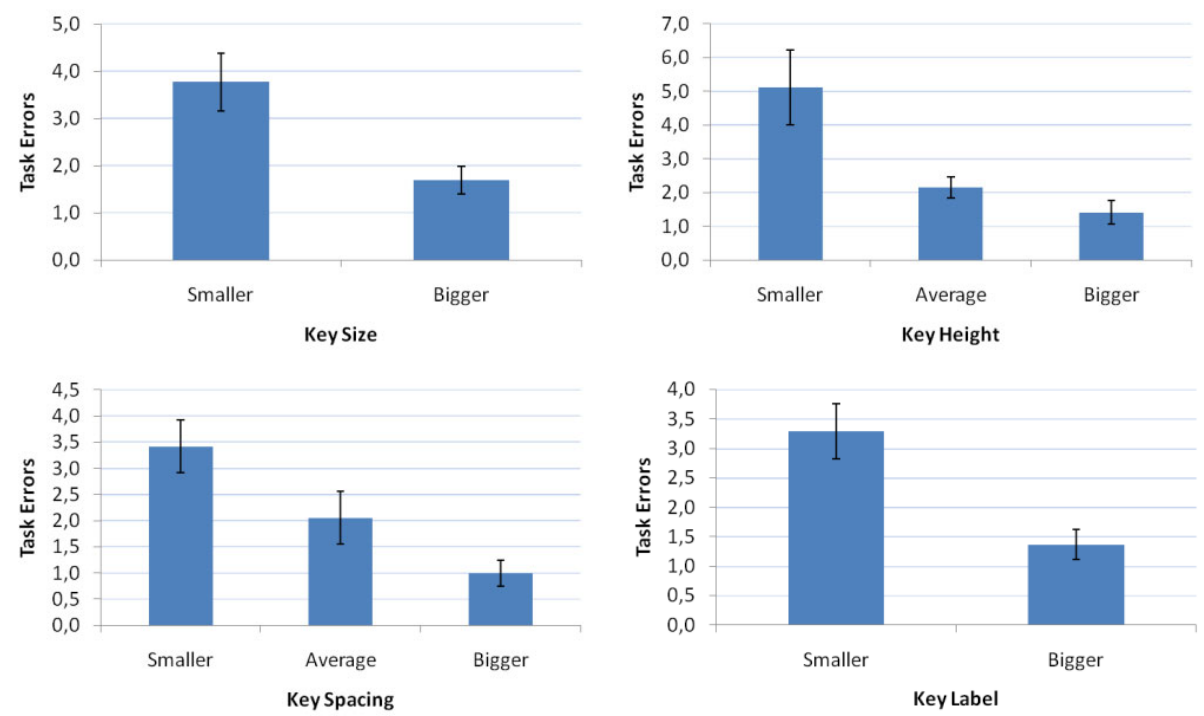

Fig. 8. Task Errors (average) for each Key Size (top left), Key Height (top right), Key Spacing (bottom left) and Key Label (bottom right). Error bars denote 95\% CI.
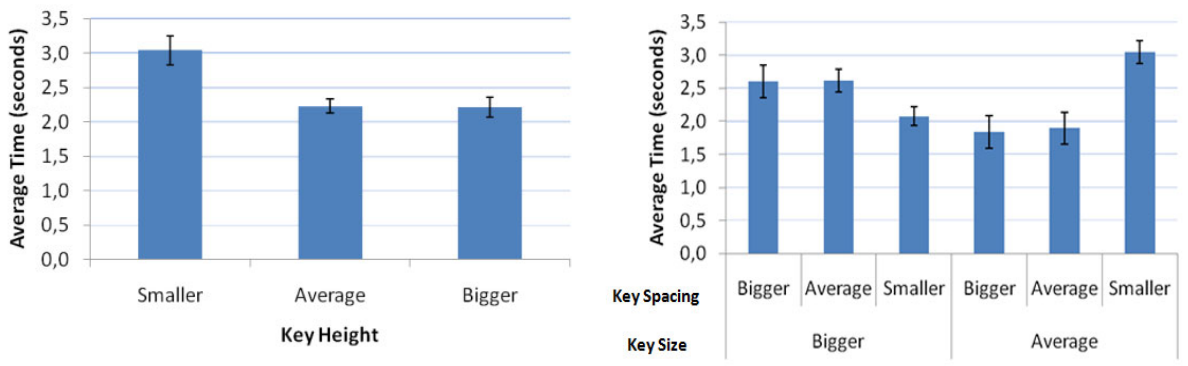

Fig. 9. Average Time for Key Height (left) and for Key Size and Spacing (right). Error bars denote $95 \%$ CI.

Key Height and Key Size had a significant effect on Task Errors $\left(F_{2,124}=3.28\right.$, $p<.05)$. Figure 10 showcases this relation, where we can observe that the devices with an average Key Size but with a small Key Height resulted in poor effectiveness. It is also apparent that independently of Key Size, the smaller the Key Height was, the more errors participants made. 

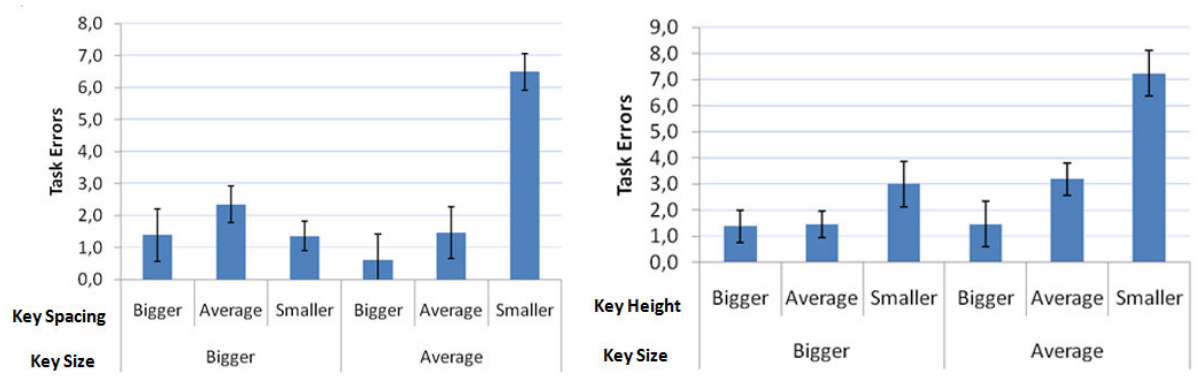

Fig. 10. Task Errors (average) for each Key Size and Key Spacing (left) and Task Errors (average) for each Key Size and Key Height (right). Error bars denote 95\% CI.

Other pair with significant impact on users' Average Time and Task Errors was Key Label and Key Spacing $\left(F_{1,125}=17.13, p<.01\right.$ and $F_{1,125}=18.70, p<.01$ respectively). A small Key Label in conjunction with a small Key Spacing, resulted in more errors and longer time than any other case, as we can see in Figure 11.

Lastly, Key Material and Key Height affected significantly $\left(F_{1,125}=19.40, p<.01\right)$ the number of errors made by the participants. Figure 11 shows that keys with a good Key Material do not have a clear benefit if they have a small Key Height, as the number of errors increased as the height of the keys decreased.
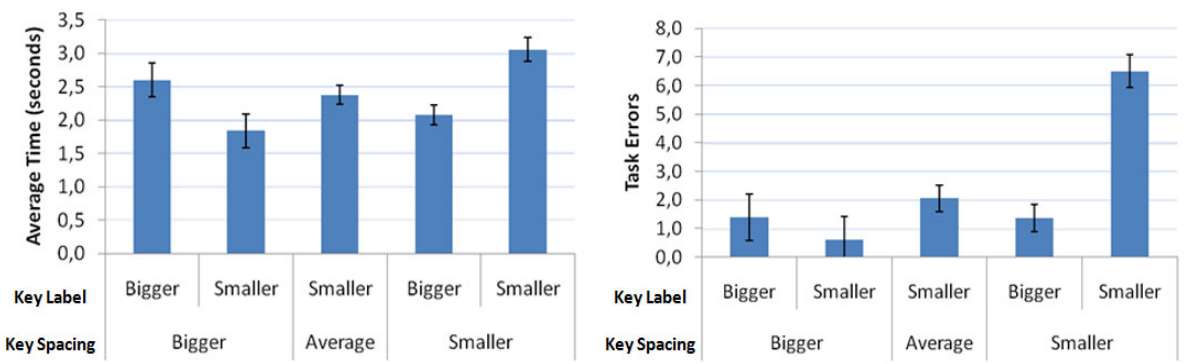

Fig. 11. Average Time for each Key Spacing and Key Label (left) and Task Errors (average) for each Key Spacing and Key Label (right). Error bars denote 95\% CI.

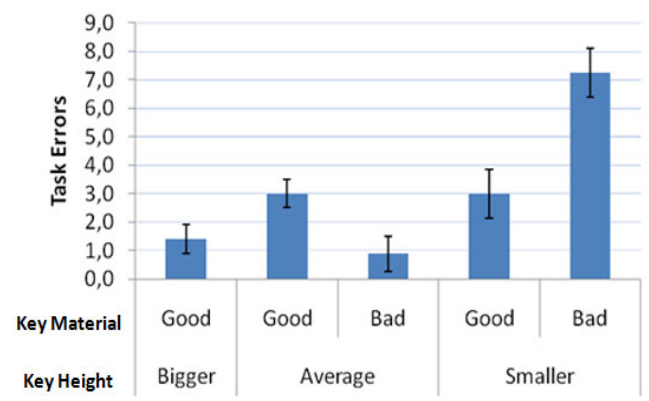

Fig. 12. Task Errors (average) for each Key Height and Key Material. Error bars denote 95\% CI. 


\section{Discussion}

Looking back to the aforementioned research questions:

1. Does tactile sensitivity affect effectiveness/efficiency on a simple key acquisition task?

Results showed that spatial acuity significantly affects the way the users acquire the keys. Users with less acuity take longer to explore the keypad and recognize the desired keys. This is more visible in tactile-wise demanding devices (low key spacing, size and relief). No significant effect was found on Task Errors. However, this could be explained with the absence of pressure as no time limits were imposed.

\section{Does cognitive ability affect effectiveness/efficiency on a simple key acquisition task?}

Verbal IQ presented a significant effect both on users' effectiveness and efficiency. The evaluations performed are suitable assessments both for attention and short-term memory. Indeed, these are abilities that are likely to change from one individual to another, particularly with age. Regarding spatial ability, no significant effects were found. This was expected as all participants were experienced with the keypad layout.

\section{Which keyboard demands have most impact on user performance?}

All the device characteristics analyzed showed to have influence on user performance. The devices present similar keypad layouts and the task is quite simple. However, differences were still found and, in some cases, dramatic. It is paramount to understand each demand and its limits. Further, this understanding goes beyond accessibility and assistive technologies. Mobile devices are challenging for everyone, every now and then, and inclusive design should be promoted for every user's benefit.

\section{Are individual differences worth considering on mobile interface design?}

Results showed that individual differences have an impact on user performance and hence they should be considered to improve effectiveness. As the tasks demands increase, likely so will the differences between users. These differences are commonly so dramatic that exclusion is reached.

\section{Conclusions}

Individual differences among the blind have a great impact on the different mobile interaction proficiency levels they attain. General-purpose interfaces and assistive technologies disregard these differences. In this paper, we argue that both the users' capabilities as the device demands should be explored to foster inclusive design. We presented a study with 13 blind people where different capability (particularly, attention and spatial acuity) levels showed to have a significant impact on key acquisition effectiveness and efficiency. It is noticeable that these differences are present even in 
the simplest task. Furthermore, mobile device demands variations showed to have a wide impact on the user's ability to interact, giving relevance to informed design.

Next, we will relate low-level user characteristics with mobile interaction modalities demands. In possession of a thorough characterization of how different users relate to different interaction modalities, we will derive a model that allows us to make predictions regarding the performance of particular user/modality pairs.

\section{References}

1. Manduchi, R., Coughlan, J.: Portable and mobile systems in assistive technology. In: Miesenberger, K., Klaus, J., Zagler, W.L., Karshmer, A.I. (eds.) ICCHP 2008. LNCS, vol. 5105, pp. 1078-1080. Springer, Heidelberg (2008)

2. Levesque, V.: Blindness, technology and haptics. Center for Intelligent Machines. Technical Report (2005)

3. Harper, S.: Standardizing electronic travel aid interaction for visually impaired people. UMIST, Technical Report (1998)

4. Leonard, R.: Statistics on Vision Impairment a Resource Manual. Lighthouse International, Technical Report (2001)

5. Hollins, M., Leung, E.: Understanding blindness: An integrative approach. Lawrence Erlbaum, Mahwah (1989)

6. Adams, A.: Electronic Travel Aids: New Directions for Research. Working Group on Mobility Aids for the Visually Impaired and Blind, Committee on Vision, NRC (1986)

7. Guerreiro, T., Jorge, J., Gonçalves, D.: Identifying the individual ingredients for a (in)successful non-visual mobile experience. In: Proceedings of the European Conference on Cognitive Ergonomics, ECCE, Delft, The Netherlands (2010)

8. Persad, U., et al.: Characterising user capabilities to support inclusive design evaluation. Universal Access in the Information Society 6(2), 119-135 (2007)

9. Guerreiro, T., et al.: NavTap: a long term study with excluded blind users. In: Assets 2009: Proceedings of the 11th International ACM SIGACCESS Conference on Computers and Accessibility, pp. 99-106. ACM, New York (2009)

10. Kane, S.K., et al.: Freedom to roam:a study of mobile device adoption and accessibility for people with visual and motor disabilities. In: Proceedings of the 11th International ACM SIGACCESS Conference on Computers and Accessibility, pp. 115-122. ACM, New York (2009)

11. Plos, O., Buisine, S.: Universal design for mobile phones: a case study. In: CHI 2006: CHI 2006 Extended Abstracts on Human Factors in Computing Systems, New York, NY, USA, pp. 1229-1234 (2006)

12. Shinohara, K., Tenenberg, J.: A blind person's interactions with technology. Commun. ACM 52(8), 58-66 (2009)

13. MacKenzie, I., Tanaka-Ishii, K.: Text entry systems: Mobility, accessibility, universality. Morgan Kaufmann, San Francisco (2007)

14. Burton, D.: You Get to Choose: An Overview of Accessible Cell Phones. Access Issues 6(2) (2005)

15. Guerreiro, T., Lagoá, P., Santana, P., Gonçalves, D., Jorge, J. NavTap and BrailleTap: Non-Visual Texting Interfaces. In: Proceedings of RESNA (2008) 
16. Kane, S., Bigham, J., Wobbrock, J.: Slide Rule: Making Mobile Touch Screens Accessible to Blind People using Multi-Touch Interaction Techniques. In: Proceedings of ASSETS, pp. 73-80 (2008)

17. Yfantidis, G., Evreinov, G.: Adaptive Blind Interaction Technique for Touchscreens. Universal Access in the Information Society 4(4), 328-337 (2006)

18. Bonner, M., Brudvik, J., Abowd, G., Edwards, W.: No-Look Notes: Accessible Eyes-Free Multitouch Text-Entry. Pervasive Computers, 409-426 (2010)

19. Gregor, P., Newell, A.F.: Designing for dynamic diversity: making accessible interfaces for older people. In: WUAUC 2001: Proceedings of the $2001 \mathrm{EC} / \mathrm{NSF}$ Workshop on Universal Accessibility of Ubiquitous Computing, pp. 90-92. ACM, New York (2001)

20. Zajicek, M.: Design principles to support older adult, pp. 111-113. Springer, Heidelberg (2004)

21. Kurniawan, S., Mahmud, M., Nugroho, Y. A Study of the Use of Mobile Phones by older Persons. In: Conference on Human Factors in Computing Systems, pp. 989-994 (2006)

22. Kurniawan, S.: Mobile Phone Design for older persons. In: Designing for Seniors: Innovations for Graying Times, pp. 24-25 (2007)

23. Czaja, S., Lee, C.: The impact of aging on access to technology. Universal Access in the Information Society 5(4), 341-349 (2007)

24. Tremblay, F., Mireault, A.C., Dessureault, L., Manning, H., Sveistrup. H. Experimental Brain Research, 155-164 (2004)

25. Mackinnon, S., Dellon, A.: Two-point discrimination tester. Journal of Hand Surgery 10A, 906-907 (1985)

26. Baddeley, A.D., Hitch, G.J.: Working memory. In: Psychology of Learning and Motivation, pp. 47-89. Academic Press, London (2000)

27. Aiken, L.R.: Assessment of Intellectual Functioning. Perspectives on Individual Differences Series. Springer, Heidelberg (2004)

28. Wechsler, D.: Wechsler Adult Intelligence Scale - Revised. Psychological Corporation, San Antonio (1981)

29. Xydias, N.: Tests pour l'orientation et la selection professionnelle des aveugles. Editions Scientifiques et Psychologiques (1977) 